\title{
VAGINAL BLEEDING IN YOUNG CHILDREN
}

\author{
Anek Aribarg and Vorapong Phupong \\ Department of Obstetrics and Gynecology, Faculty of Medicine, Chulalongkorn University, \\ Bangkok, Thailand
}

\begin{abstract}
To evaluate prospectively vaginal bleeding in young children with emphasis on the cause, management and outcome, we analyzed the data of all children aged under 10 years old who presented with vaginal bleeding at the King Chulalongkorn Memorial Hospital between 1981 and 2000. There were 55 girls presenting with vaginal bleeding with variable degrees of severity. In 41 of the 55 girls (74.5\%), the bleeding resulted from a local lesion of the genital tract. Genital tumors were seen in four girls, ie hemangioma of the vulva, sarcoma botryoides of the vagina, functional ovarian cyst and granulosa cell tumor of the ovary. In the remaining 14 girls (25.5\%), the bleeding originated from systemic hormonal etiology. In one girl with precocious puberty, it was associated with hypothyroidism, whereas in the other six girls it was due to constitutional causes. In addition, the cause of bleeding in 10 girls was the vaginal manifestation of leeches, which is a unique problem for Thailand. Although vaginal bleeding in young children is rare, it can be an alarming clinical presentation, and serious underlying causes should be excluded. Prompt and correct diagnosis will lead to successful management.
\end{abstract}

\section{INTRODUCTION}

Vaginal bleeding in young children is an alarming symptom. Because there may be a serious underlying causes, such as genital tumors, all children should be carefully investigated and correctly managed. We prospectively collected the etiology of vaginal bleeding in girls aged under 10 years who were under our management at the King Chulalongkorn Memorial Hospital, Bangkok, Thailand, between 1981 and 2000, and compared them with previous reports. Ten years of age was chosen as the upper limit for this study, because after this time the bleeding may have a physiological cause (Dewhurst, 1971; Reiter and Kulin, 1972).

\section{MATERIALS AND METHODS}

The hospital records of all children, 10

Correspondence: Dr Vorapong Phupong, Department of Obstetrics and Gynecology, Faculty of Medicine, Chulalongkorn University, Rama IV Road, Pathumwan, Bangkok 10330, Thailand.

Tel: 66 (0) 2256 4241; Fax: 66 (0) 22549292

E-mail: vorapong.p@chula.ac.th years of age and under, who presented with vaginal bleeding and were managed during the period 1981-2000, were collected. Investigations of the children included detailed medical history, and physical and genital examination. Special laboratory and other investigations were made when it was appropriate, including hematological, hormonal, ultrasound, X-ray and other sophisticated examinations. History taking was done to determine the etiology of the bleeding, its duration and the manifestation of any cyclical pattern. Clinical examination was conducted to record secondary sexual characteristics, if present. Most of the children were examined under general anesthesia. The final diagnosis, subsequent management and followup results were also noted.

\section{RESULTS}

In total, 55 girls were seen during the study period; their ages ranged from newborn to 10 years. The etiology of the vaginal bleeding is shown in Table 1. The most common cause of vaginal bleeding was trauma from an accident. In 41 girls (74.5\%), the bleeding resulted from a local lesion, and in $4(7.3 \%)$ 
Table 1

Etiology of vaginal bleeding in young children, King Chulalongkorn Memorial Hospital.

\begin{tabular}{clrc}
\hline \multicolumn{1}{c}{ Etiology } & Number $(\%)$ & Age (years) \\
\hline Aroup & Hormonal withdrawal bleeding & & \\
& Newborn & $6(10.9)$ & Newborn \\
& Accidental pill ingestion & $1(1.8)$ & 4 \\
B & Trauma & $17(30.9)$ & Newborn-9 \\
& Accidental & $5(9.1)$ & $6-9$ \\
C & Rape & $6(10.9)$ & $7-9$ \\
& Sexual precosity & $1(1.8)$ & 6 \\
& Constitutional (idiopathic) & $4(7.3)$ & $4-9$ \\
D & Hypothyroidism & $2(3.6)$ & $6-8$ \\
E & Tumor & $1(1.8)$ & 9 \\
F & Foreign body & $1(1.8)$ & 7 months \\
H & Infection & $10(18.2)$ & $5-9$ \\
I & Arteriovenous malformation & 1 & 5 \\
& Leech & $55(1.8)$ & \\
\hline
\end{tabular}

of them, the bleeding was from genital tract tumors. Where no local lesion was demonstrable, the bleeding of the remaining $14(25.5 \%)$ girls was associated with some form of precocious puberty or hormonal withdrawal symptom. One case of precocious puberty was associated with hypothyroidism, while the remaining six cases were diagnosed as constitutional causes. Seventeen children had vaginal bleeding caused by accidental falls, while five children were sexually assaulted. We found two cases of vaginal foreign body.

The genital tract tumors causing vaginal bleeding were one case each of hemangioma of the vulva, functional ovarian cyst, granulosa cell tumor of the ovary and sarcoma botryoides of the vagina. The case of sarcoma botryoides was managed with radical surgery followed by combined chemotherapy; the girl died six months after treatment.

One girl, aged seven months, had vaginal bleeding as a result of uterine arteriovenous malformation. Ten children aged between 5 and 10 years had vaginal bleeding from a leech entering their vagina while swimming in a river or pond. In one child, the bleeding was so severe that a blood transfusion was required.

The range of follow-up period in this study was 6 months to 2 years.

\section{DISCUSSION}

When vaginal bleeding occurs in children, two groups of conditions should be considered. In the first, the bleeding is caused by a vulvae or vaginal lesion. In the second, the bleeding arises from the endometrium itself, usually as a manifestation of premature puberty (Baldwin and Landa, 1995; Muram, 1990). Diagnosis and treatment require a detailed examination (Cowan and Morrison, 1991; Muram, 1990). An adequate inspection of the perineum can be achieved if the child is in the frog-leg position. At times, the knee-chest position is more favorable for examination. If the examination cannot be performed easily, or if the upper vagina must be examined to exclude trauma or tumor, examination should be conducted under general anesthesia. Vaginoscopy with small diameter instruments allows adequate endoscopic examination of the vaginal 
Table 2

Comparison of causes of vaginal bleeding.

\begin{tabular}{|c|c|c|c|c|}
\hline Cause & $\begin{array}{c}\text { Imai et al } \\
\quad(1996) \\
\mathrm{N}=62\end{array}$ & $\begin{array}{c}\text { Hill et al } \\
(1989) \\
\mathrm{N}=52\end{array}$ & $\begin{array}{c}\text { Heller et al } \\
(1978) \\
\mathrm{N}=51\end{array}$ & $\begin{array}{l}\text { Present study } \\
\qquad \mathrm{N}=55\end{array}$ \\
\hline Vulvovaginitis & $45.2 \%$ & $6 \%$ & $6 \%$ & $1.8 \%$ \\
\hline Genital tumor & $14.5 \%$ & $21 \%$ & $12 \%$ & $9.1 \%$ \\
\hline Transitory & $4.8 \%$ & - & - & - \\
\hline Urethral prolapse & $9.7 \%$ & $10 \%$ & - & $1.8 \%$ \\
\hline Trauma & $9.7 \%$ & $8 \%$ & $8 \%$ & $40 \%$ \\
\hline Foreign body & $4.8 \%$ & - & - & $3.6 \%$ \\
\hline Vulvar lesion & - & $10 \%$ & - & - \\
\hline Vaginal prolapse & - & - & $2 \%$ & - \\
\hline Unknown & $11.3 \%$ & $25 \%$ & - & - \\
\hline Hormonal withdrawal & - & - & - & $12.7 \%$ \\
\hline Leech & - & - & - & $18.2 \%$ \\
\hline Precocious puberty & $14.5 \%$ & $21 \%$ & $72 \%$ & $12.7 \%$ \\
\hline Idiopathic & $6.4 \%$ & $3.8 \%$ & $29 \%$ & $10.9 \%$ \\
\hline McCune Albright syndrome & - & - & $17 \%$ & - \\
\hline Precocious menstruation & $8.1 \%$ & $11.5 \%$ & $16 \%$ & - \\
\hline CNS lesion & - & - & $10 \%$ & - \\
\hline Hypothyroid & - & - & - & $1.8 \%$ \\
\hline Granulosa cell tumor & - & $5.7 \%$ & - & - \\
\hline
\end{tabular}

CNS: central nervous system.

canal and cervix. If peritoneal entry is suspected, laparotomy is indicated (Cowan and Morrison, 1991; Muram, 1990).

Genital injury due to rape or sexual molestation requires special legal and social procedures, which vary from country to another. However, careful examination, detailed records and repair of the laceration under general anesthesia are essential medical management.

We found $74.5 \%$ of the cases had local causes of bleeding similar to the report by Imai et al (1995); our findings, however, are in contrast with other reports (Heller et al, 1978; Hill et al, 1989), which found $27-54 \%$ (Table 2).

In our series, vaginal bleeding in children was most frequently associated with trauma, in 22 out of 55 girls (40\%), which is contradictory to some other series (Cowan and Morrison, 1991; Fishman and Paldi, 1991; Heller et al, 1978; Hill et al, 1989; Imai et al, 1995; Muram, 1990), which reported only $8-9.7 \%$.
The most common cause of vaginal bleeding in our study was trauma, while vulvovaginitis was the most common cause of vaginal bleeding in previous studies (Cowan and Morrison, 1991; Fishman and Paldi 1991; Imai et al, 1995; Muram, 1990), which reported 45-75\%. However, we found only one in 55 cases $(1.8 \%)$ of vulvovaginitis. One report showed that malignant genital tumors and precocious puberty were the most common etiology (Hill et al, 1989). Anothers report from Heller et al (1978) found idiopathic precocious puberty the most common cause of vaginal bleeding. The difference may be attributed to differences in race, habits of the people, local conditions and the types of hospital practice, and whether they were general or specialized.

During childhood, vaginal bleeding should alert us to the possibility of a genital tumor or foreign body. Reported on malignant tumors of the genital tract were sarcoma botryoides, adenocarcinoma, and endodermal sinus tumor of the vagina (Imai et al, 1995). Heller et al 
(1978) reported $12 \%$ of vaginal bleeding in young children attributable to genital tumors. In our series, there were four cases $(7.3 \%)$ of genital tract tumors. They consisted of hemangioma of the vulva, functional ovarian cyst, granulosa cell tumor of the ovary, and sarcoma botryoides of the vagina. We managed the cases of hemangioma of the vulva and functional ovarian cyst conservatively, and they disappeared spontaneously. We managed the case of granulosa tumor of the ovary with conservative surgery similar to that of Sanfilippo and Wakim (1987). In addition, the case of sarcoma botryoides of the vagina underwent radical surgery, followed by combined chemotherapy similar to previous reports (Muram, 1990; Sanfilippo and Wakim, 1987). Unfortunately, the girl died six months after treatment. Previous suggestions (Cowan and Morrison, 1991) showed that biopsy should be performed in the case of genital tumors.

Other vaginal bleeding occasionally resulted from urethral prolapse (Imai et al, 1995; Muram, 1990). Ninety-nine percent of cases appear before 10 years of age or after 50 years of age (Fishman and Paldi, 1991). Urethral prolapse is a circular eversion of the mucosa of urethra at the meatus (Baldwin and Landa, 1995; Fishman and Paldi, 1991; Hill et al, 1989; Sanfilippo and Wakim, 1987). Predisposing factors were coughing, constipation, trauma, urinary and vaginal infections (Fernandes et al, 1993). This condition can be easily diagnosed when the mass is seen at the urethral orifice (Imai et al, 1995). There were three methods used for therapy: conservative management (consisted of sitz bath, local application of estrogen cream, and oral antibiotics) (Baldwin and Landa, 1995; Fernandes et al, 1993; Fishman and Paldi, 1991; Sanfilippo and Wakim, 1987), ligation over a Foley catheter (Fernandes et al, 1993), and total excision of the prolapse (Fernandes et al, 1993; Sanfilippo and Wakim, 1987). In the series, we found only one $(1.8 \%)$ with this lesion. We managed our case by excision and had no recurrence during a two-year follow-up period.

Seventeen cases $(30.9 \%)$ in our series bled from accidental trauma; some cases falls were from bicycles, and the girls were injured by the handle-bars. The remaining five cases $(9.1 \%)$ bled from sexual molestation. However, Heller et al (1978) reported 8\% of cases were accidental trauma. Most of the cases were caused by falling onto sharp objects (Fishman and Paldi, 1991). Examination under general anesthesia is always necessary because of the laceration of the vulva, which might appear relatively insignificant from external inspection, and the external blood loss might seem minute in the presence of significant vaginal hematoma (Heller et al, 1978; Hill et al, 1989) and vaginal rupture.

Foreign bodies are another cause of vaginal bleeding. We identified 3.6\% in the series, in contrast to other series that reported $4.8 \%$ (Imai et al, 1995), and were not mentioned in other reports (Heller et al, 1978; Hill et al, 1989). The most common foreign body in our cases, and some other previous reports, were rolled pieces of toilet paper (Baldwin and Landa, 1995; Fishman and Paldi, 1991; HermanGiddens, 1994; Muram, 1990; Sanfilippo and Wakim, 1987), which may appear as grayish masses (Baldwin and Landa, 1995). The classical triad of symptoms are vaginal bleeding or spotting, discharge and odor (HermanGiddens, 1994). Treatment is with viscous lidocaine, followed by the insertion of a 10or $12-\mathrm{F}$ catheter, and lavage with a diluted povidone-iodine solution to wash out the foreign body (Baldwin and Landa, 1995).

Other causes of vaginal bleeding, were uterine arteriovenous malformation (AVM) and a leech entering the vagina. We found one case of AVM of the uterus in a 7-month-old infant, presenting with severe vaginal bleeding. We made the diagnosis by angiogram, the gold standard for diagnosis (Hoffman et al, 1997). After recurrent episodes of severe bleeding, we decided to perform a hysterectomy to control the bleeding. A leech entering the vagina is a common cause of vaginal bleeding in the tropics. We found a large number of cases (10 cases). We had previously reported and proposed a guideline for the management of this 
condition (Aribarg and Phupong, 1999). If the bleeding ceases after a short period of observation, no further treatment is required. If the bleeding persists, the vagina should be examined and irrigated with physiological normal saline solution. Then the bleeding point should be cleaned thoroughly to ensure that the leech's saliva is completely removed. Then, the vagina should be firmly packed with gauze, which is to be removed after 24 hours. The vaginal bleeding caused by leeches is a problem unique to Thailand and other tropical countries.

Another source of vaginal bleeding was from the endometrium, a manifestation of precocious puberty. We found seven cases $(12.7 \%)$ of precocious puberty; six were idiopathic or constitutional, while one was due to hypothyroidism. The findings are similar to those reported by Imai et al (1995), of 14.5\%; however, it is in contrast to the report of Heller et al (1978), of 73\%. Our case and previous reports (Gordon et al, 1997; Rakover et al, 1993) demonstrated vaginal bleeding in premenarcheal girls was caused by hypothyroidism, which was diagnosed by clinical manifestation and confirmed by the levels of serum thyroxine and thyroid stimulating hormone, and successful treatment with thyroxine.

Seven cases bled from hormonal withdrawal. Six of them were newborn. One case was the result of an accidental ingestion of pills. This may be explained by the fact that the infant girls respond physiologically to the stimulation of placentally acquired maternal estrogen during their intrauterine life (Baldwin and Landa, 1995; Muram, 1990). Careful history taking will be helpful for diagnosis. We managed these cases with reassurance of the parents and elimination of the exogenous estrogen source, similar to the previous suggestion by Sanfilippo and Wakim (1987).

In conclusion, vaginal bleeding in children is a rare condition. If it occurs, a serious underlying cause should be excluded. Prompt and precise examination, combined with correct management will usually lead to cure and preservation of the girl's future reproductivity.

\section{REFERENCES}

Aribarg A, Phupong V. Vaginal bleeding in Thai children due to the leech. J Trop Med Parasitol 1999; 22: 77-9.

Baldwin DD, Landa HM. Common problems in pediatric gynecology. Urol Clin North Am 1995; 22 : 161-76.

Cowan BD, Morrison JC. Management of abnormal genital bleeding in girls and women. $N$ Engl $J$ Med 1991; 324: 1710-5.

Dewhurst J. Gynaecological disease in childhood. Update 1971; 3: 549-70.

Fernandes ET, Dekermacher S, Sabadin MA, Vaz F. Urethral prolapse in children. Urology 1993; 41: 240-3.

Fishman A, Paldi E. Vaginal bleeding in premenarcheal girls: a review. Obstet Gynecol Surv 1991; 46: 457-60.

Gordon CM, Austin DJ, Radovick S, Laufer MR. Primary hypothyroidism presenting as severe vaginal bleeding in a prepubertal girl. J Pediatr Adolesc Gynecol 1997; 10: 35-8.

Heller ME, Savage MO, Dewhurst J. Vaginal bleeding in childhood: a review of 51 patients. $\mathrm{Br} J$ Obstet Gynaecol 1978; 85: 721-4.

Herman-Giddens ME. Vaginal foreign bodies and child sexual abuse. Arch Pediatr Adolesc Med 1994; 148: 195-200.

Hill NCW, Oppenheimer LW, Morton KE. The aetiology of vaginal bleeding in children: a 20-year review. Br J Obstet Gynaecol 1989; 96: 467-70.

Hoffman MK, Meilstrup JW, Shackelford DP, Kaminski PF. Arteriovenous malformations of the uterus: an uncommon cause of vaginal bleeding. Obstet Gynecol Surv 1997; 52: 736-40.

Imai A, Horibe S, Tamaya T. Genital bleeding in premenarcheal children. Int J Gynaecol Obstet 1995; 49: 41-5.

Muram D. Vaginal bleeding in childhood and adolescence. Obstet Gynecol Clin North Am 1990; 17 : 389-408.

Rakover Y, Weiner E, Shalev E, Luboshitsky R. Vaginal bleeding: presenting symptom of acquired primary hypothyroidism in a seven year-old girl. J Pediatr Endocrinol 1993; 6: 197-200.

Reiter EO, Kulin HE. Sexual maturation in the female. Normal development and precocious puberty. Pediatr Clin North Am 1972; 19: 581-603.

Sanfilippo JS, Wakim NG. Bleeding and vulvovaginitis in the pediatric age group. Clin Obstet Gynecol 1987; 30: 653-61. 